\title{
Left Anterior Temporal Lobe and Bilateral Anterior Cingulate Cortex Are Semantic Hub Regions: Evidence from Behavior-Nodal Degree Mapping in Brain-Damaged Patients
}

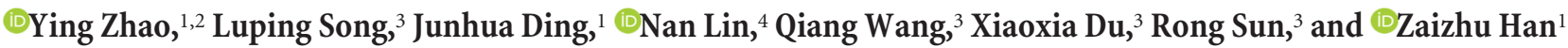 \\ ${ }^{1}$ State Key Laboratory of Cognitive Neuroscience and Learning and International Data Group/McGovern Institute for Brain Research, Beijing Normal \\ University, Beijing 100875, China, ${ }^{2}$ Neuroscience and Aphasia Research Unit, School of Psychological Sciences, University of Manchester, Manchester M13 \\ 9PL, United Kingdom, ${ }^{3}$ Rehabilitation College and China Rehabilitation Research Center, Capital Medical University, Beijing 100038, China, and ${ }^{4}$ CAS Key \\ Laboratory of Behavioral Science, Institute of Psychology, Beijing 100101, China
}

The organizational principles of semantic memory in the human brain are still controversial. Although studies have shown that the semantic system contains hub regions that bind information from different sensorimotoric modalities to form concepts, it is unknown whether there are hub regions other than the anterior temporal lobe (ATL). Meanwhile, previous studies have rarely used network measurements to explore the hubs or correlated network indexes with semantic performance, although the most direct supportive evidence of hubs should come from the network perspective. To fill this gap, we correlated the brain-network index with semantic performance in 86 brain-damaged patients. We especially selected the nodal degree measure that reflects how well a node is connected in the network. The measure was calculated as the total number of connections of a given node with other nodes in the resting-state functional MRI network. Semantic ability was measured using the performance of both general and modality-specific (object form, color, motion, sound, manipulation, and function) semantic tasks. We found that the left ATL and the bilateral anterior cingulate cortex could be semantic hubs because the reduced nodal degree values of these regions could effectively predict the deficits in both general and modality-specific semantic performance. Moreover, the effects remained when the analyses were performed only in the patients who did not have lesions in these regions. The two hub regions might support semantic representations and executive control processes, respectively. These data provide empirical evidence for the distributed-plus-hub theory of semantic memory from the network perspective.

Key words: brain-damaged patient; graph-theoretical network analysis; lesion- behavior correlation; semantic hub; semantic network

Significance Statement

Although the distributed-plus-hub organization of semantic memory has been proposed for several years, it remains unclear which hubs other than the anterior temporal lobe are included in the semantic system. Here, we identified such hubs from an innovative network perspective. The voxelwise nodal degree values were correlated with the performance of general and modalityspecific semantic tasks in 86 patients with brain damage. We observed that the left anterior temporal lobe and bilateral anterior cingulate cortex could be semantic hubs because their decreased nodal degree values were significantly correlated with the severity of the deficit in semantic performance. The two hub regions might contribute to semantic representational and control processes, respectively. These findings offer new evidence for the distributed-plus-hub theory.

\section{Introduction}

Semantic memory refers to general world knowledge about objects, words, people, and facts (Tulving, 1972). Although re- searchers have reached a consensus that semantic knowledge is represented in a distributed neural network in the human brain (Patterson et al., 2007; Binder and Desai, 2011; Han et al.,
Received June 16, 2016; revised Oct. 13, 2016; accepted Nov. 11, 2016.

Author contributions: Y.Z., L.S., N.L., and Z.H. designed research; Y.Z., L.S., J.D., N.L., Q.W., X.D., and R.S. performed research; Y.Z., J.D., N.L., and Z.H. analyzed data; Y.Z., L.S., J.D., and Z.H. wrote the paper.

This work was supported by the 973 Program (2013CB837300, 2014CB846100), the National Natural Science Foundation of China (31271115, 31571129, 81171019), the Major Project of the National Social Science Foundation (13\&ZD188), and the Fundamental Research Funds for the Central Universities (2014kJCA07). We thank CNLab members for data collection and imaging preprocessing. We are also grateful to all research participants for their patience.
The authors declare no competing financial interests.

Correspondence should be addressed to Zaizhu Han, State Key Laboratory of Cognitive Neuroscience and Learning and IDG/McGovern Institute for Brain Research, Beijing Normal University, Beijing 100875, China. E-mail: zzhhan@bnu.edu.cn.

DOI:10.1523/JNEUROSCI.1946-16.2016

Copyright $\odot 2017$ the authors $\quad 0270-6474 / 17 / 370141-11 \$ 15.00 / 0$ 
2013a,b; Martin, 2016; Huth et al., 2016), they hold different opinions about the organizational principles of the network. There have been at least two prevalent theories for this issue: the distributed-only theory and the distributed-plus-hub theory (Patterson et al., 2007, 2015; Mahon and Caramazza, 2009; Lambon Ralph et al., 2010; Martin et al., 2014; Martin, 2016). The former postulates that a semantic concept is represented by modality-specific sensorimotor knowledge (e.g., shape, color, sound, and action; Martin, 2007; Barsalou, 2008; Binder et al., 2009). Meanwhile, the latter argues that the semantic system includes hubs in addition to the modality-specific knowledge, and it is the hubs that form semantic concepts by binding information from different modalities (Patterson et al., 2007; Lambon Ralph et al., 2010). In this case, a hub should process information from various modalities, and the disruption of this component leads to semantic decline across modalities. Researchers are coming close to resolving this debate (Lambon Ralph, 2014; Rice et al., 2015a,b). They have observed that at least the anterior temporal lobe (ATL) could be one semantic hub region. A piece of supportive evidence for this notion comes from studies on semantic dementia (Patterson et al., 2007). This disease is a progressive neurodegenerative disorder characterized by the selective loss of semantic memory, regardless of sensorimotoric modalities (Hodges et al., 1992; Garrard and Hodges, 2000; Hodges and Patterson, 2007; Patterson et al., 2007; Gorno-Tempini et al., 2011). The cognitive symptom of semantic dementia is associated with damage to the ATL (Hodges et al., 1992; Patterson et al., 2007; Binney et al., 2010; Gainotti, 2012; Hoffman et al., 2014; Lambon Ralph, 2014; Rice et al., 2015a,b). The role of the ATL as a semantic hub has also been demonstrated by other studies, including those in other disease patients (Patterson et al., 2007; Tsapkini et al., 2011; Lambon Ralph et al., 2012; Shimotake et al., 2015) and in healthy subjects (Pobric et al., 2007, 2010; Lambon Ralph et al., 2009; Visser et al., 2010; Peelen and Caramazza, 2012; Chiou and Lambon Ralph, 2016).

Although the ATL has been proven to be a semantic hub region, it is still uncertain whether there are other semantic hubs in the brain. Studies have reported other possible semantic hub regions, such as the anterior cingulate cortex (ACC), the fusiform gyrus, the intraparietal sulcus, the posterior temporal middle gyrus, and the prefrontal cortex (Jefferies and Lambon Ralph, 2006; Binder et al., 2009; Mion et al., 2010; Binder and Desai, 2011; Wei et al., 2012; Guo et al., 2013; Seghier, 2013; Bonnici et al., 2016). However, the observed hub effects might warrant further confirmation because these studies only considered the outcomes of healthy participants, the effects of local regions in isolation but not in networks, or the cerebral indexes alone without correlating with the semantic behaviors. Thus, further research should investigate the relationships between the network index and semantic behavior in a patient group.

The current study was designed to determine the presence of other semantic hub regions in addition to the ATL by correlating the hub property of each voxel in a whole-brain resting-state functional MRI (rs-fMRI) network with the severity of semantic deficits in 86 brain-damaged patients. The hub property was measured using the nodal degree, which is an index of graph-theory analysis and can reflect how well connected a node is in the whole-brain network. The semantic behaviors were measured by two sets of tasks: general semantic tasks and modality-specific semantic tasks. A region was con- sidered a semantic hub if its nodal degree could successfully predict the scores of both sets of semantic tasks.

\section{Materials and Methods \\ Participants}

Healthy subjects and patients with brain damage took part in the current study. Most of them were selected from the subject cohort of our recent study (Han et al., 2013b). The differences in subject populations between the studies were due to the availability of behavioral and neuroimaging data. All participants were native Chinese speakers, were provided written informed consent, and were paid for their participation. This study was approved by the Institutional Review Board of the State Key Laboratory of Cognitive Neuroscience and Learning, Beijing Normal University.

\section{Patients}

Eighty-six adults with brain damage (69 males) were recruited from the China Rehabilitation Research Center (mean age, 44 years; SD, 13; range, 19-74 years). They had their first brain injury with more than 1 month post-onset (mean, 5.4 months; SD, 11.2; range, 1-86 months), had no other neurological or psychiatric diseases, had at least a primary school education (mean, 13 years; SD, 3; range, 6-19 years), and could understand our task instructions. The Edinburgh Handedness Inventory (Oldfield, 1971) revealed that most of the subjects were right-handed $(n=80)$; five were left-handed and one was ambidextrous. The causes of brain injury included stroke $(n=69)$, traumatic brain injury (TBI; $n=$ $14)$, anoxic encephalopathy $(n=1)$, electric shock $(n=1)$, and thrombosis $(n=1)$. The brain lesions of the stroke patients were widely distributed in gray-matter cortices but were concentrated in the bilateral insula, whereas those of the TBI patients mainly included ventral and polar ATL regions (Fig. 1).

\section{Healthy subjects}

Fifty-one healthy individuals (27 males) without brain injury were included. The mean age was 50 years (SD, 11; range, $26-72$ years), and the mean years of formal education was 13 (SD, 4; range, 6-22 years). All were right-handed. They had normal or corrected-normal vision and hearing and had no history of psychiatric or neurological disease.

Compared with the healthy subjects, the patient cohort was younger $(t=2.55, p<0.02)$ and had more males $\left(\chi^{2}=11.37, p=0.0008\right)$, but the groups had an equivalent educational level $(t=0.23, p=0.82)$.

\section{Behavioral data collection}

To measure the subjects' ability to process various semantic knowledge, each subject was examined by two sets of semantic tasks: general and modality-specific tasks (Table 1). The former set included three tasks: oral picture naming, oral sound naming, and picture associative matching. All of these tasks tested the general aspect of semantic memory but varied in the modalities of input and output. The latter set assessed the processing ability for six specific sensorimotor modalities of objects (form, color, motion, sound, manipulation, and function). Each modality was examined by verbal and nonverbal tasks. We also designed a calculation task to control for the influence of nonsemantic processing.

All tasks were presented with the DMDX display program (Forster and Forster, 2003). Participants were tested individually in a quiet room. Each session lasted $\leq 2 \mathrm{~h}$, and pauses for rest were allowed upon request. The presentation orders in each task were pseudorandomized but were identical across subjects. We calculated the composite score of each set of tasks-the general semantic memory and modality-specific semantic memory tasks — and defined them as indexes of the patients' general and modality-specific semantic memory, respectively.

\section{General semantic tasks}

Oral picture naming. The oral picture-naming task $(n=100)$ used 100 photographs of objects from five categories: animals, tools, artifacts, fruits and vegetables, and large nonmanipulable objects. Each category 

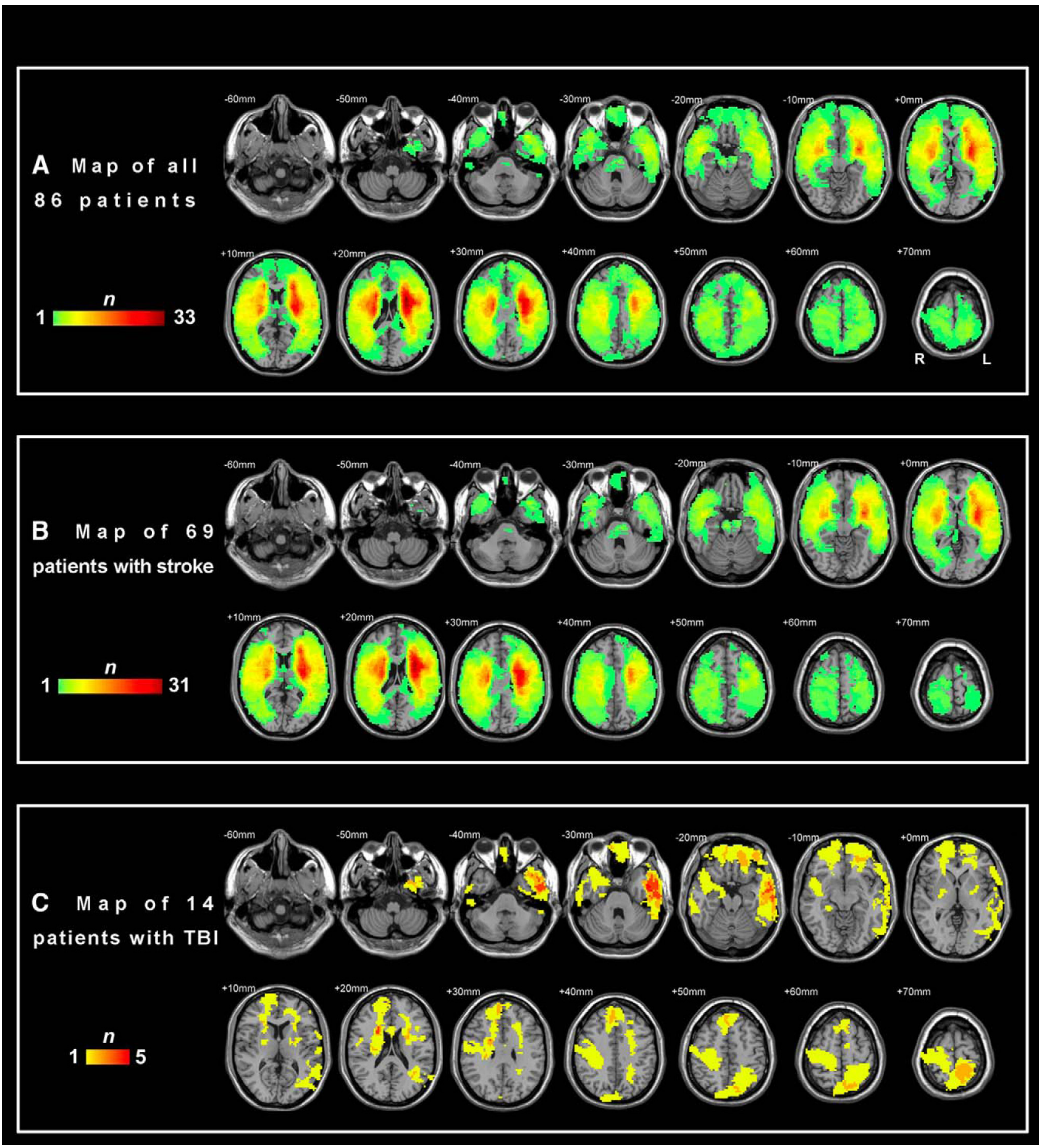

Figure 1. A-C, Lesion overlap maps of patients (the $n$ value denotes the number of patients with lesions). TBI, traumatic brain injury.

contained 20 items. Participants were instructed to name the object shown on the computer screen.

Oral sound naming. The oral sound-naming task $(n=36)$ included 10 animal sounds (e.g., a barking dog), six tool sounds (e.g., pounding of a hammer), 10 sounds from man-made artifacts (e.g., ringing of a telephone), and 10 other sounds (e.g., a sound of rain). Participants listened to the sounds through earphones and then had to name the objects that produced the sounds.

Picture associative matching. The picture associative matching task $(n=50)$ is similar to the Pyramids and Palm Trees Test (Howard and Patterson, 1992). For each trial, three photographs are presented in a triangle. Participants need to determine which one of the two bottom photographs (e.g., lion, tadpole) was semantically closer to the top one (frog). The number of items was identical across the five categories in the above oral picture-naming task.

Verbal modality-specific tasks

Each sensorimotor modality was tested with a verbal task and a nonverbal task. Each trial of the verbal task was visually presented with a question about a given sensorimotor modality (e.g., which one has four legs?) with two candidate object words (e.g., panda, peacock) on a touch screen. To rule out the influence of dyslexia, the experimenter also read the stimuli aloud to the participants. Participants needed to 
Table 1. Behavioral performance of subjects

\begin{tabular}{|c|c|c|c|}
\hline \multirow[b]{2}{*}{ Tasks } & \multicolumn{2}{|c|}{ Raw accuracy: mean (SD) } & \multirow{2}{*}{$\begin{array}{l}\text { Corrected } \\
\text { t score (SD) } \\
\text { of patients }\end{array}$} \\
\hline & Healthy controls & Patients & \\
\hline \multicolumn{4}{|l|}{ General semantic task } \\
\hline Oral picture naming & $96 \%(3 \%)$ & $76 \%(27 \%)^{* * *}$ & $-6.87(8.63)$ \\
\hline Oral sound naming & $81 \%(11 \%)$ & $58 \%(27 \%)^{* * *}$ & $-2.67(2.57)$ \\
\hline Picture associative matching & $94 \%(4 \%)$ & $88 \%(10 \%)^{* * *}$ & $-1.52(2.33)$ \\
\hline \multicolumn{4}{|l|}{ Modality-specific semantic task } \\
\hline \multicolumn{4}{|l|}{ Verbal task } \\
\hline Form attribute matching & $94 \%(5 \%)$ & $87 \%(12 \%)^{* * *}$ & $-1.55(2.41)$ \\
\hline Color attribute matching & $95 \%(5 \%)$ & $85 \%(13 \%)^{* * *}$ & $-2.36(3.08)$ \\
\hline Motion attribute matching & $93 \%(5 \%)$ & $85 \%(11 \%)^{* * *}$ & $-1.79(2.21)$ \\
\hline Sound attribute matching & $86 \%(9 \%)$ & $78 \%(13 \%)^{* * *}$ & $-1.03(1.49)$ \\
\hline Manipulation attribute matching & $93 \%(5 \%)$ & $82 \%(15 \%)^{* * *}$ & $-2.55(3.04)$ \\
\hline Function attribute matching & $98 \%(3 \%)$ & $91 \%(14 \%)^{* * *}$ & $-2.76(5.66)$ \\
\hline \multicolumn{4}{|l|}{ Nonverbal task } \\
\hline Form verification & $83 \%(9 \%)$ & $75 \%(13 \%)^{* * *}$ & $-1.28(1.49)$ \\
\hline Color verification & $79 \%(12 \%)$ & $64 \%(16 \%)^{* * *}$ & $-1.38(1.34)$ \\
\hline Motion verification & $69 \%(15 \%)$ & $54 \%(18 \%)^{* * *}$ & $-1.37(1.23)$ \\
\hline Sound verification & $85 \%(8 \%)$ & $73 \%(14 \%)^{* * *}$ & $-1.80(1.68)$ \\
\hline Manipulation judgment & $90 \%(8 \%)$ & $81 \%(14 \%)^{* * *}$ & $-1.26(1.67)$ \\
\hline Function judgment & $96 \%(4 \%)$ & $90 \%(12 \%)^{* * *}$ & $-1.40(2.52)$ \\
\hline \multicolumn{4}{|l|}{ Control task } \\
\hline Number calculation & $95 \%(11 \%)$ & $80 \%(26 \%)^{* * *}$ & $-1.05(2.36)$ \\
\hline
\end{tabular}

choose the object that was the better answer to the question. The tasks and examples are described as follows.

Form attribute matching. In the form attribute matching task $(n=75)$, an example question is: Which has four legs, cat or goldfish?

Color attribute matching. In the color attribute matching task $(n=30)$, an example question is: Which one is brownish, lion or penguin?

Motion attribute matching. In the motion attribute matching task $(n=$ 30), an example question is: Which one moves faster, monkey or panda?

Sound attribute matching. In the sound attribute matching task $(n=$ 45), an example question is: Whose sound is sharper, a monkey's or a cow's?

Manipulation attribute matching. In the manipulation attribute matching task $(n=30)$, an example question is: Which of the following is manipulated with one hand, chopsticks or a carpenter's plane?

Functional attribute matching. In the functional attribute matching task $(n=60)$, an example question is: Which one is more common in people's home, an electric hair dryer or an electric drill?

\section{Nonverbal modality-specific tasks}

All trials of the nonverbal modality-specific tasks were presented with pictures, movies, or sounds without verbal input or output.

Form verification. For each form verification trial $(n=60)$, two grayscale photographs of object parts (e.g., head of panda, body of bear) were presented vertically at the center of a touch screen. The subjects had to determine whether the two parts came from the same object.

Color verification. Each color verification trial $(n=20)$ consisted of two pictures: a color patch (e.g., green patch) at the top and a gray-scale object (horse) at the bottom. Participants needed to verify whether the color was common for the object.

Motion verification. Each motion verification trial $(n=57)$ included a video of a dot moving as an object (e.g., the running of horse) and a gray-scale picture of an object (cow). The task was to verify whether the movement was a typical motion for the pictured object.

Sound verification. Each sound verification trial $(n=42)$ included a gray-scale picture of an object (e.g., monkey) and the sound of an object (the meow of a cat). The task was to decide whether the sound was typically produced by the object in the picture.

Manipulation matching. This manipulation matching task $(n=20)$ was similar to the above picture associative matching task, except the instruction was changed to choose which of the two bottom objects (e.g., clamp, fork) was more similar to the top object (a pair of tweezers) in terms of the typical manner of manipulation.
Function matching. The function matching task $(n=30)$ was same as the above manipulation matching task, except the instruction was replaced with the judgment of which object in the two bottom photographs (e.g., clock, bag) shared more similar functions with the object in the top photograph (watch).

\section{Control task}

Number processing has been extensively used to control for semantic processing profiles (Pobric et al., 2007; Lambon Ralph et al., 2009; Binney et al., 2010; Han et al., 2013b). Therefore, a number calculation task was adopted in this study. It contained seven exact calculation questions: two additions $(5+2,19+26)$, two subtractions $(9-4,78-15)$, two multiplications $(2 \times 4,13 \times 6)$, and one division $(6 \div 2)$. The subjects were required to give correct answers for each question presented on the screen.

Some of the above tasks were also used in our recent studies (Han et al., 2013a,b; Bi et al., 2015; Fang et al., 2015).

\section{Behavioral data preprocessing}

The above tasks could be classified into three different types according to the manner in which subjects responded: oral production, matching, and verification tasks. Responses were made by oral reporting, pressing the corresponding object, and pressing a "YES" or "NO" button, respectively. Scoring for each trial was performed on the first complete response for the oral production tasks, and on the first response for the other tasks. Each subject obtained a raw accuracy score for each task.

Given that the patients had varied demographic characteristics (age, gender, and education level), their raw scores might not truly reflect the severity of the deficits in behavioral performance. Therefore, we adopted a standardization method proposed by Crawford and Garthwaite (2006) to correct each patient's "raw" score to a standardized " $t$ " score by considering the distribution of the 51 healthy subjects. This method has been used in our recent studies (Han et al., 2013b; Bi et al., 2015; Fang et al., 2015). Eventually, the raw accuracy of each task for each patient was transformed into a corrected $t$ score (Crawford and Garthwaite, 2006; Han et al., 2013b).

For each patient, we further computed a composite general semantic score (by averaging $z$-transformed $t$ scores of the three general semantic tasks) and a composite modality-specific semantic score (by averaging $z$-transformed $t$ scores of all the six modality tasks). The two semantic composite scores and the $t$ score of the control task were adopted in the following analyses.

\section{Neuroimaging data collection}

We collected three types of images using a $1.5 \mathrm{~T}$ General Electric Signa Excite scanner at the China Rehabilitation Research Center: (1) highresolution 3D T1-weighted images, (2) rs-fMRI images, and (3) fluid attenuated inversion recovery (FLAIR) T2-weighted images. The 3D images were obtained on the sagittal plane with the following parameters: matrix size, $512 \times 512$; voxel size, $0.49 \times 0.49 \times 0.70 \mathrm{~mm}^{3}$; $\mathrm{TR}=12.26$ $\mathrm{ms}$; TE $=4.2 \mathrm{~ms}$; inversion time, $400 \mathrm{~ms}$; flip angle, $15^{\circ}$; slice thickness, $0.70 \mathrm{~mm}$; slice gap, $0.70 \mathrm{~mm}$; field of view, $250 \times 250 \mathrm{~mm}^{2}$; slice number, 248. Two identical sequences of the 3D T1 images were collected and averaged to improve the signal-to-noise ratio for analysis. The rs-fMRI images were acquired using an EPI sequence along the AC-PC line with the following parameters: $\mathrm{TR}=2000 \mathrm{~ms}$; $\mathrm{TE}=40 \mathrm{~ms}$; flip angle, $90^{\circ}$; slice thickness, $4 \mathrm{~mm}$; slice gap, $1 \mathrm{~mm}$; voxel size, $3.3 \times 3.3 \times 4.0 \mathrm{~mm}^{3}$; FOV, $210 \times 210 \mathrm{~mm}^{2}$; slice number, 28 ; volume number, 120 . During the scanning, participants were instructed to keep still with their eyes closed and not to think about anything in particular. The FLAIR images that had the same slice locations with the functional images on the axial plane were acquired with the following parameters: matrix size, $512 \times 512$; voxel size, $0.49 \times 0.49 \times 0.70 \mathrm{~mm}^{3}$; $\mathrm{TR}=8002 \mathrm{~ms}$; TE $=127.57 \mathrm{~ms}$; flip angle, $90^{\circ}$, slice thickness, $5 \mathrm{~mm}$; voxel size, $0.49 \times 0.49 \times 5.00 \mathrm{~mm}^{3}$; FOV, $250 \times 250 \mathrm{~mm}^{2}$; slice number, 28 .

\section{Neuroimaging data preprocessing}

\section{Structural MRI data}

The preprocessing procedures were identical to those used in our recent studies (Han et al., 2013b; Fang et al., 2015). We coregistered the two T1 
images using SPM5 and then averaged them. Then, we coregistered the FLAIR T2 images and resliced them to the averaged T1 images. Two trained personnel drew each patient's lesion contour based on the averaged 3D images slice by slice, visually referring to the corresponding FLAIR T2 images. The lesion maps were double-checked by an experienced radiologist. Then, each patient's structural images were resliced to a $1 \times 1 \times 1 \mathrm{~mm}^{3}$ voxel size and manually registered into Talairach space via the "3D Volume Tools" in BrainVoyager QX v2.0 (www. brainvoyager.com). We used ANTs software to estimate the affine transformation matrix between the native and Talairach spaces. With this matrix, we transformed the lesion maps into Talairach space using the "WarpImageMultiTransform" program. The lesion maps were then transformed into MNI space for further analysis.

\section{rs-fMRI data}

Each member of the patient group had a brain lesion large enough to possibly affect the quality of normalization if we had used automated software. Therefore, we manually coregistered each patient's restingstate scans to individual T1 images in BrainVoyager. Other preprocessing steps were performed with the advanced edition of Data Processing Assistant for Resting-State fMRI (DPARSFA; Yan and Zang, 2010). In detail, the preprocessing procedure included (1) time point deletion (the first 10 volumes); (2) slice timing; (3) head-motion correction; (4) manual coregistration of images to the averaged 3D T1 images followed by transformation into Talairach space using the affine transformation matrix obtained in the structural MRI data preprocessing procedure; and (5) transformation of images into the MNI space. The remaining steps were also completed with DPARSFA, including (6) liner trend removal, (7) bandpass filtering $(0.01-0.1 \mathrm{~Hz}),(8)$ spatial smoothing (Gaussian kernel of FWHM $6 \mathrm{~mm}$ ), and (9) nuisance covariate (global signal, white-matter signal, and CSF signal) regression. Specifically, we then performed head-motion scrubbing (Power et al., 2012) to exclude brain volumes that had spikes of head motion. We set the framewise displacement threshold at 0.5 to detect the "bad" time points and scrubbed one time point before and two time points after the bad time point. In addition, a subject would be excluded from our analyses if his or her head motion exceeded $3 \mathrm{~mm}$ translation or $3^{\circ}$ rotation. Because no subject reached the exclusion criterion, all of them entered into the following analyses.

\section{Extracting the weighted nodal degree value of each voxel}

Using the preprocessed rs-fMRI data, we calculated the weighted voxelwise nodal degree for each patient with GRETNA (Graph Theoretical Network Analysis Toolbox; http://www.nitrc.org/projects/gretna/; Wang et al., 2015). Specifically, we first computed the strength of the functional connectivity of each pair of voxels, which was the Pearson correlation coefficient $(r)$ between the rs-fMRI signal intensity time courses of the two voxels. Then we obtained the nodal degree value of each voxel by summing the values of all functional connections connecting to the voxel in the whole-brain gray-matter network. The gray-matter mask we used was generated by including voxels with a probability of $>0.4$ in the SPM5 gray-matter template and excluding voxels that fell on cerebellar regions defined by the Automated Anatomical Labeling template (Tzourio-Mazoyer et al., 2002). The final gray-matter mask had 36,272 voxels.

\section{Identifying semantic hubs}

To identify the hubs in semantic networks, each of the semantic composite scores (general semantic scores or modality-specific semantic scores) was separately correlated with the nodal degree value of each voxel across patients. Then, the obtained results were converged.

\section{General semantic scores-nodal degree correlation}

We correlated the nodal degree value of each voxel with general semantic composite scores and partialled out the corrected $t$ score of the calculation task. AlphaSim correction was used to adjust the influence of multicomparisons (corrected $p<0.05$; voxel level: $p<0.05$; cluster size, $>110$ voxels).
To further confirm that the observed effects originated from the connections of the cluster rather than the lesions located in the cluster, we again performed the above partial correlational analyses for each detected cluster within the subgroup of patients who had no lesion in each cluster. To conduct these analyses for each cluster, we first extracted the patients who had no lesion there. Then, each patient's mean degree values in the cluster were calculated. Finally, the values were correlated with general semantic composite scores, factoring out the corrected $t$ score of the calculation task.

\section{Modality-specific semantic scores-nodal degree correlation}

This analysis was the same as that of the above general semantic scores correlation, except that the general semantic composite scores were replaced with the modality-specific semantic composite scores.

\section{Convergent analysis of both of the above results}

Each region that reached the significance threshold in either of the above analyses could potentially be a semantic hub. However, to avoid falsepositive results, we conducted a convergent analysis for the results of the two analyses. We extracted the common voxels of the above two results and then considered the regions of common voxels to be semantic hubs.

\section{Validation of the observed effects by covarying the influence of} clinical variables

Although the above analyses ruled out the influence of demographic variables by correcting behavioral performance (age, gender, educational level), they did not account for the potential confounding effects of the clinical factors (e.g., etiology, lesion size, postonset duration). To validate whether the semantic-hub association effects we observed could be accounted for by these clinical factors, we performed partial correlations between patients' mean nodal values of each hub region and the composite general semantic scores or modality-specific semantic scores, regressing out brain lesion type (a binary variable: 1 for stroke, 2 for TBI), total lesion volume (total number of lesioned voxels in the whole brain), and disease duration (months of brain damage), in addition to the corrected $t$ score of the calculation task. Six patients were excluded from this analysis due to inexact onset time or other brain lesion types. The same analysis was also conducted on the patients without lesions in the regions of interest. The regions showing significant effects in these analyses were identified as semantic hubs.

\section{Results}

\section{Behavioral performance of participants}

Participants' raw accuracies and corrected $t$ scores are presented in Table 1. The raw accuracies of the patients (mean, 77\%) were significantly lower than those of the control group (mean, 89\%) in each task $(p<0.001)$. The corrected $t$ scores of patients $(<-1.05)$ also reflected that they had marked impairments in the tasks. For the 12 sensorimotor modality tasks, we performed a two-factor variance analysis of 2 stimuli attributions (verbal, nonverbal) $\times 6$ sensorimotor modalities (form, color, motion, sound, manipulation, and function). It revealed significant main effects (stimuli attribution: $F=8.18, p<0.005$; sensorimotor modality: $F=3.55, p<0.004)$ and interaction effects $(F=8.46$, $p<0.0001)$.

\section{Semantic hub regions}

The mean and SD values of the nodal degrees of patients are illustrated in Figure 2. The bilateral precuneus/posterior cingulate gyri, inferior parietal cortices/angular gyri, and medial prefrontal regions showed higher mean degree values.

\section{General semantic scores-nodal degree correlation}

To find semantic hubs, we conducted a voxelwise correlational analysis between the general semantic composite scores and the nodal degree value across 86 patients, with the corrected $t$ score of the number calculation task as a covariate. We observed five regions that survived the significance threshold (AlphaSim- 

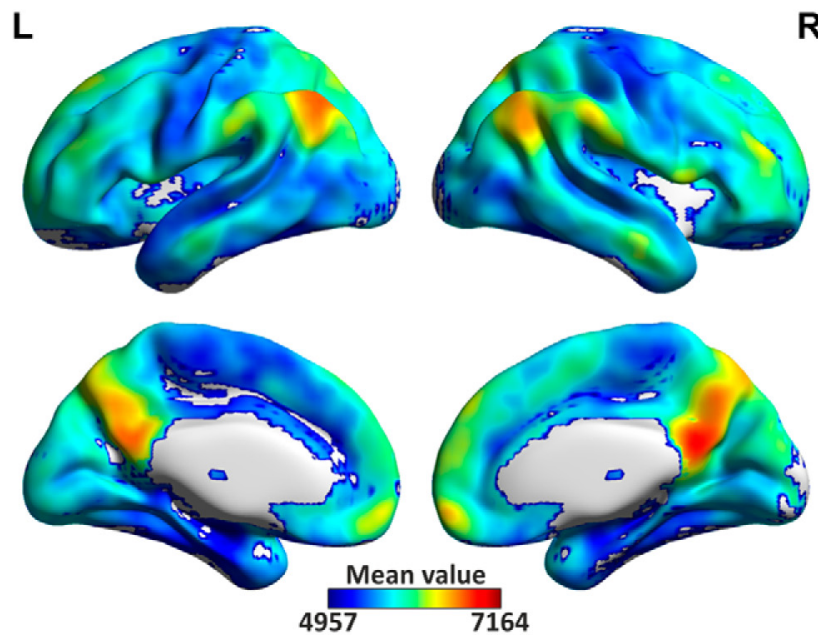

$\mathbf{R}$

$\mathbf{L}$
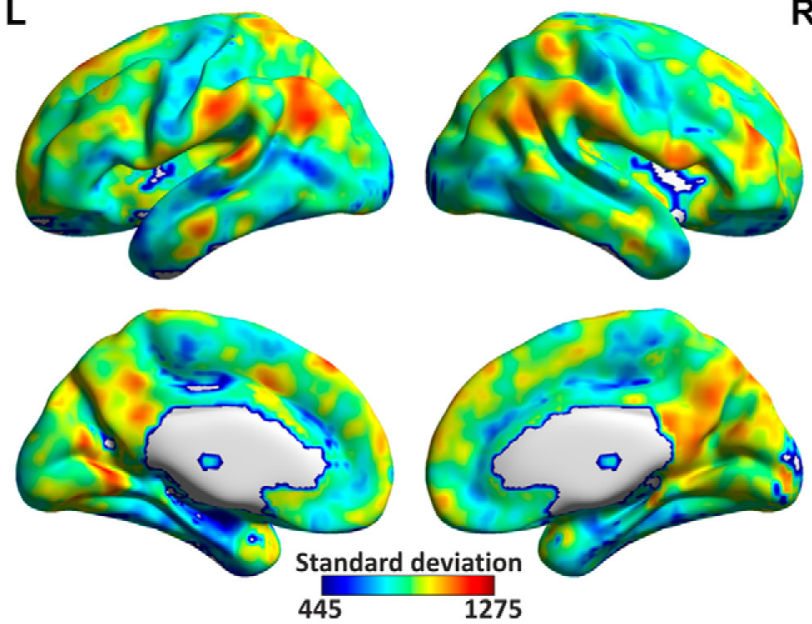

Figure 2. Mean and standard deviation values of the voxelwise nodal degrees on patients. $L$, left; $R$, right.

corrected $p<0.05$; Table 2; Fig. $3 A$ ). The correlations were positive in four regions: the bilateral ACC (coordinates peak $:-9,48$, $12 ; r_{\text {peak }}=0.40, p<0.0002$; cluster size, 346 voxels; $r_{\text {cluster }}=0.39$, $p<0.0003$ ), the left ATL (coordinates peak $_{1}:-60,-18,-15$; $r_{\text {peak }}=0.35, p<0.002$; cluster size, 328 voxels; $r_{\text {cluster }}=0.46, p<$ $0.0001)$, the left superior parietal lobule (SPL; coordinates peak: : $-24,-63,60 ; r_{\text {peak }}=0.38, p<0.0004$; cluster size, 126 voxels; $\left.r_{\text {cluster }}=0.37, p<0.0005\right)$, and the left middle frontal gyrus

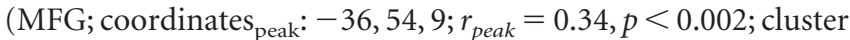
size, 120 voxels; $\left.r_{\text {cluster }}=0.37, p<0.0005\right)$. The bilateral precuneus showed a negative correlation (coordinates peak $_{\text {: }}-18,-69$, $18 ; r_{\text {peak }}=-0.39, p<0.0003$; cluster size, 254 voxels; $r_{\text {cluster }}=$ $-0.33, p<0.003)$.

The number of patients with lesions were not equivalent in the five regions observed above (Table 2). Twenty-five patients had lesions in ATL. Meanwhile, eight patients had lesions in the left MFG, five had lesions in the bilateral ACC, five had lesions in the bilateral precuneus, and four had lesions in the left SPL. For each region, we again calculated the correlation between the semantic composite scores and the mean nodal degree values of the region (averaging the degree values of all voxels in the region), covarying the corrected $t$ scores of the control task, across the patients without lesions in the region. We found that the correlations of the five regions remained significant $\left(r_{\text {sub }}:-0.31,0.31\right.$ to $0.44, p<$ $0.007)$, indicating that the observed effects of the regions might come from their functional connectivity.

\section{Modality-specific semantic scores-nodal degree correlation}

We found two clusters whose nodal degree values were significantly correlated with the modality-specific composite semantic scores when the control task scores were partialled out (AlphaSim-corrected $p<0.05$; Table 2; Fig. 3B): the bilateral ACC (coordinates peak $: 0,36,-6 ; r_{\text {peak }}=0.36, p<0.0008$; cluster size, 630 voxels; $\left.r_{\text {cluster }}=0.43, p<0.0001\right)$ and the left ATL (extending to insular, coordinates peak $:-42,6,0 ; r_{\text {peak }}=0.33, p<$ 0.002 ; cluster size, 337 voxels; $\left.r_{\text {cluster }}=0.44, p<0.0001\right)$. Eight of the 86 patients had lesions in the bilateral ACC, and 35 patients had lesions in the left ATL. In the remaining patients, the correlation effects remained significant for the two clusters (bilateral ACC: $r_{\text {sub }}=0.42, p<0.0001$; left ATL: $r_{\text {sub }}=0.35, p<0.02$; Table 2).

Convergent regions in both of the above analyses

To obtain the common brain regions for the above two analyses, each observed cluster was first created as a binary mask. Then, the five masks of the general semantic scores were overlaid onto the two masks of the modality-specific semantic scores. This process generated two common regions (Fig. $3 C$; Table 2): the bilateral ACC (center coordinates: -3, 36, 12; cluster size, 248 voxels) and the left ATL (center coordinates: -48, 0, -27; cluster size, 137 voxels).

For the common clusters, we also performed partial correlational analyses within the subjects who had no lesions in each cluster. Four of the 86 patients had lesions in the bilateral ACC and 21 patients had lesions in the left ATL. In the remaining patients, the correlation effects remained significant for the two regions (bilateral ACC-general semantics: $r_{\text {sub }}=0.37, p<$ 0.0007; bilateral ACC-modality-specific semantics: $r_{\text {sub }}=0.38$, $p<0.0005$; left ATL-general semantics: $r_{\text {sub }}=0.42, p<0.0007$; left ATL-modality-specific semantics: $\left.r_{\text {sub }}=0.42, p<0.0007\right)$.

Validation of the observed effects by covarying the influence of clinical variables

The two brain lesion types of patients (stroke, TBI; Fig. 1) had significant difference in the total lesion volume (stroke patients: mean, 2232 voxels; SD, 2175; TBI patients: mean, 977 voxels; SD, 926; $t=3.73, p<0.001$ ), but no difference in the postonset time (stroke patients: mean, 3.17 months; SD, 4; TBI patients: mean, 13.36 months; SD, 25; $t=-1.45, p=$ 0.17 ), the general semantic composite scores (stroke patients: mean, -0.03 ; SD, 0.86 ; TBI patients: mean, 0.16 ; SD, $0.74 ; t=$ $-0.77, p=0.45)$ and the modality-specific semantic composite scores (stroke patients: mean, -0.06 ; SD, 0.72; TBI patients: mean, $0.25 ; \mathrm{SD}, 0.62 ; t=-1.47, p=0.15)$. The total lesion volume was not significantly correlated with the postonset time $(r=-0.21, p=0.85)$ and the general semantic composite scores $(r=-0.18, p=0.11)$, but was correlated with the modality-specific semantic composite scores $(r=$ $-0.24, p<0.03)$. The postonset time was not significantly correlated with the two semantic composite scores (general semantics: $r=0.09, p=0.41$; modality-specific semantics: $r=$ $0.13, p=0.24)$. However, it significantly correlated with the voxelwise nodal degree values in bilateral parietal lobes, inferior temporal gyri, superior frontal gyri, the right inferior frontal gyrus, the left MFG, and the cuneus (AlphaSimcorrected $p<0.05)$. When factoring out the influence of the three clinical variables (brain lesion type, total lesion volume, postonset month) and the control task (number calculation), the composite general or modality-specific semantic scores 
Table 2. Clusters whose degree values were significantly correlated with semantic composite scores

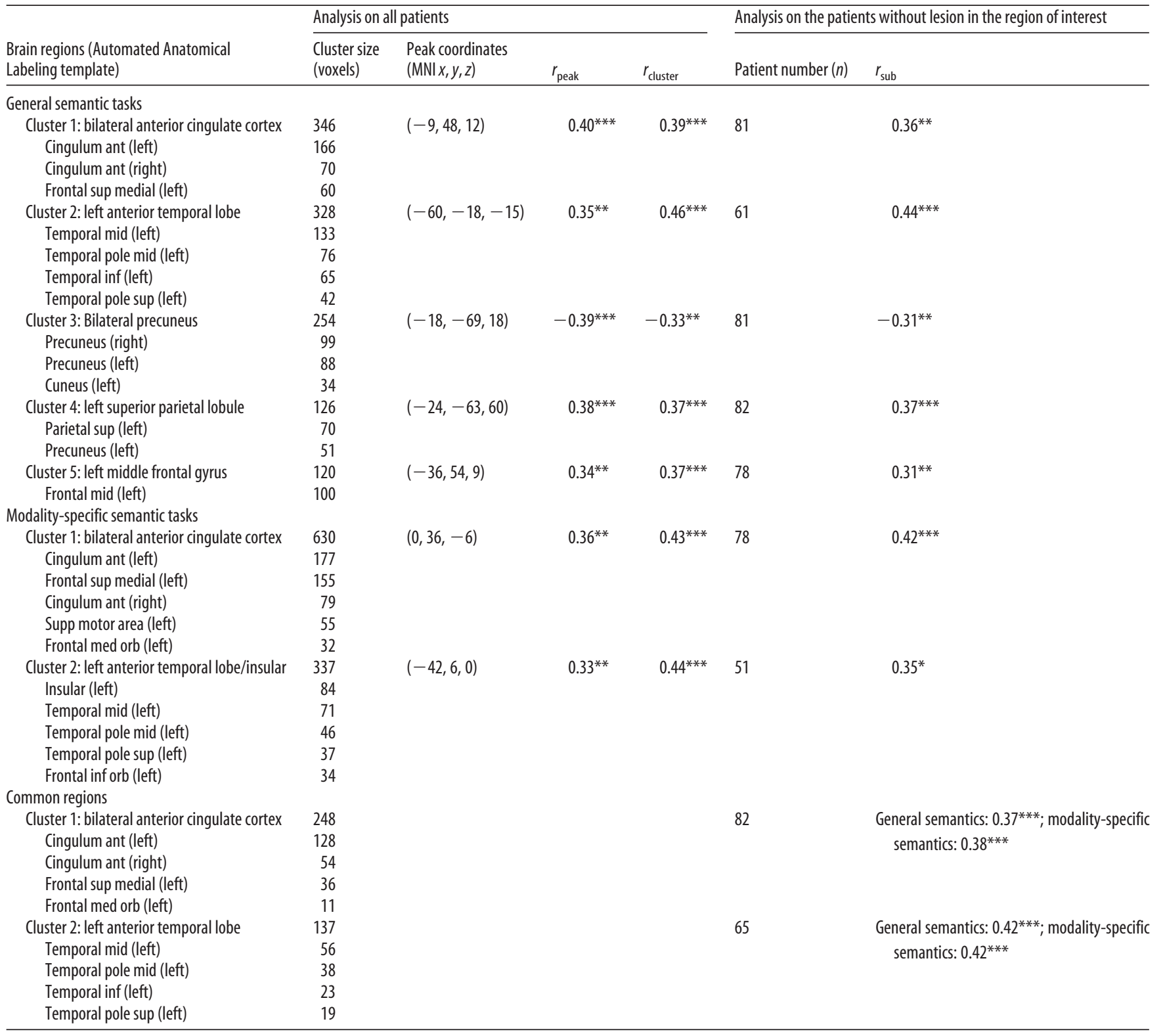

${ }^{*} p<0.05 ;{ }^{* *} p<0.01 ;{ }^{* * *} p<0.001 . r_{\text {peak }}$ Partial correlation coefficient between the degree in the peak voxel and behavioral scores; $r_{\text {cluster }}$ partial correlation coefficient between the mean nodal degree within the region and the behavioral scores; $r_{\text {sub }}$, partial correlation coefficient between the mean nodal degree within the region and the behavioral scores among the patients who had no lesion in the corresponding cluster. For simplicity, only the clusters with $>30$ voxels are listed for the general and modality-specific tasks.

remained significantly correlated with the mean nodal values of both observed regions across the 80 patients (bilateral ACC-general semantics: $r=0.38, p<0.0007$; bilateral ACCmodality-specific semantics: $r=0.39, p<0.0006$; left ATL-general semantics: $r=0.45, p<0.0001$; left ATLmodality-specific semantics: $r=0.42, p<0.0002)$. A similar pattern was also observed in the analysis for the patients without lesions in the observed hub regions. Seventy-seven patients were analyzed for the bilateral ACC and 59 for the left ATL. The partial correlation values were as follows: bilateral ACC-general semantics: $r_{\text {sub }}=0.40, p<0.0005$; bilateral ACC-modality-specific semantics: $r_{\text {sub }}=0.40, p<0.0006$; left ATL-general semantics: $r_{\text {sub }}=0.40, p<0.004$; left ATLmodality-specific semantics: $r_{\text {sub }}=0.39, p<0.004$.

The above analyses demonstrated that the left ATL and bilateral ACC could be critical hubs for semantic memory because the disconnection of these regions causes dysfunction in semantic processing.

\section{Exploring the role of the right anterior temporal lobe in semantic processing}

The semantic hubs we identified did not include the right ATL, even though it has been widely reported to be engaged in semantic processing in local analyses (Visser et al., 2010). One possible reason for this seeming contradiction is that the region is primarily responsible for nonverbal rather than verbal semantic information (Rice et al., 2015a). To test this idea, we separately correlated the mean nodal degree values of this region mask with the composite scores in verbal tasks or nonverbal tasks by averaging $z$-transformed $t$ scores of verbal tasks or nonverbal tasks, respectively. As a control region, the left ATL hub was subjected to the same analysis. The right ATL mask was created by reversing the mask of the left ATL hub region we found in the above analyses (Fig. $3 C$ ). We found that the right ATL was marginally significantly correlated with nonverbal semantics $(r=0.21, p=$ $0.054)$ but not correlated with verbal semantics $(r=0.05, p=$ $0.65)$. In contrast, the effects of the left ATL were still significant 

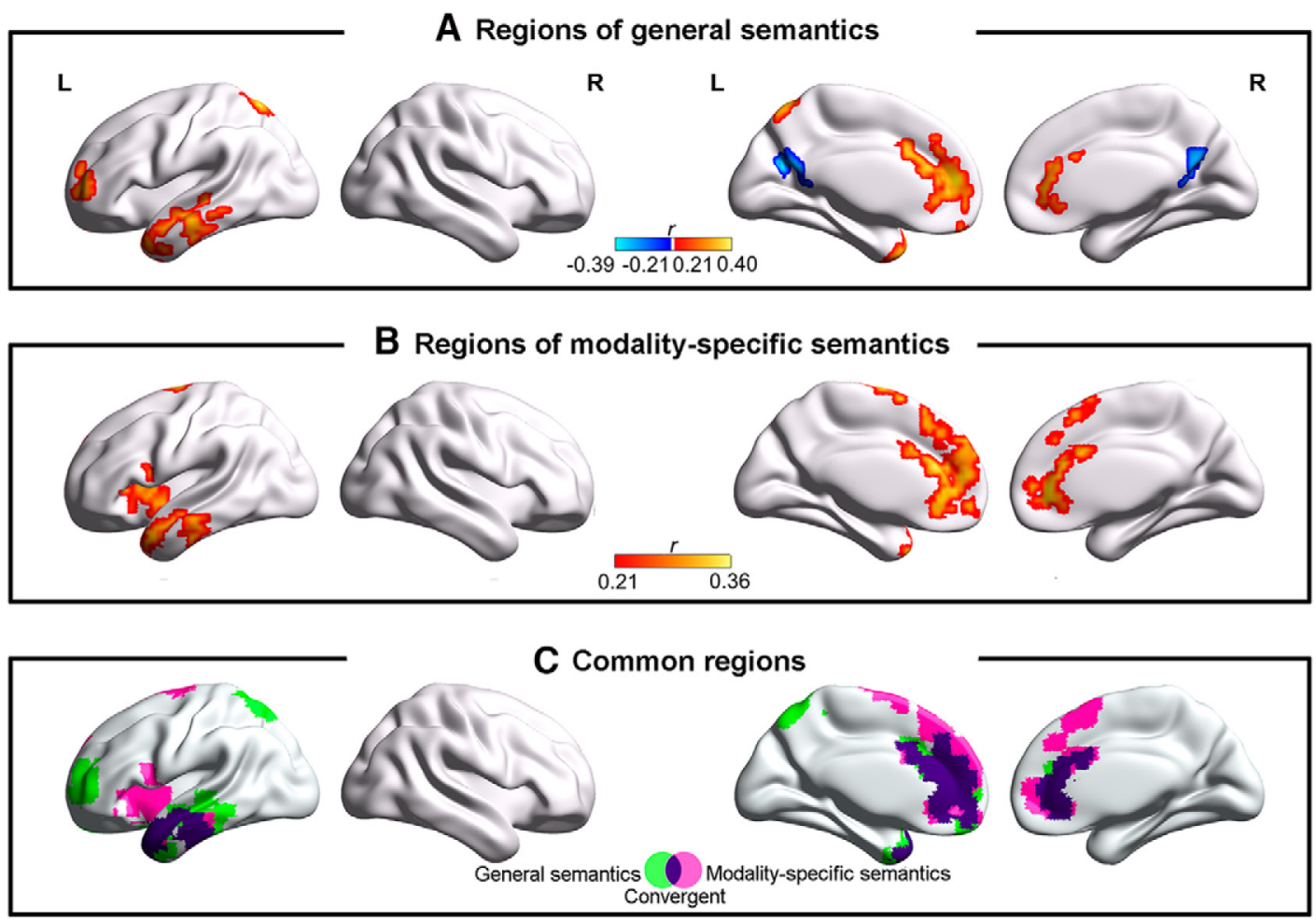

Figure 3. Correlations between nodal degree values and semantic performance across patients. $A$, Voxel nodal degree and general semantic composite score mapping. $\boldsymbol{B}$, Voxel nodal degree and modality-specific semantic composite score mapping. Regions were significant at AlphaSim-corrected $p<0.05$ (voxel level: $p<0.05$; cluster size, $>110$ ). C, Convergent results of the above two maps. See detailed information for the regions in Table 2.
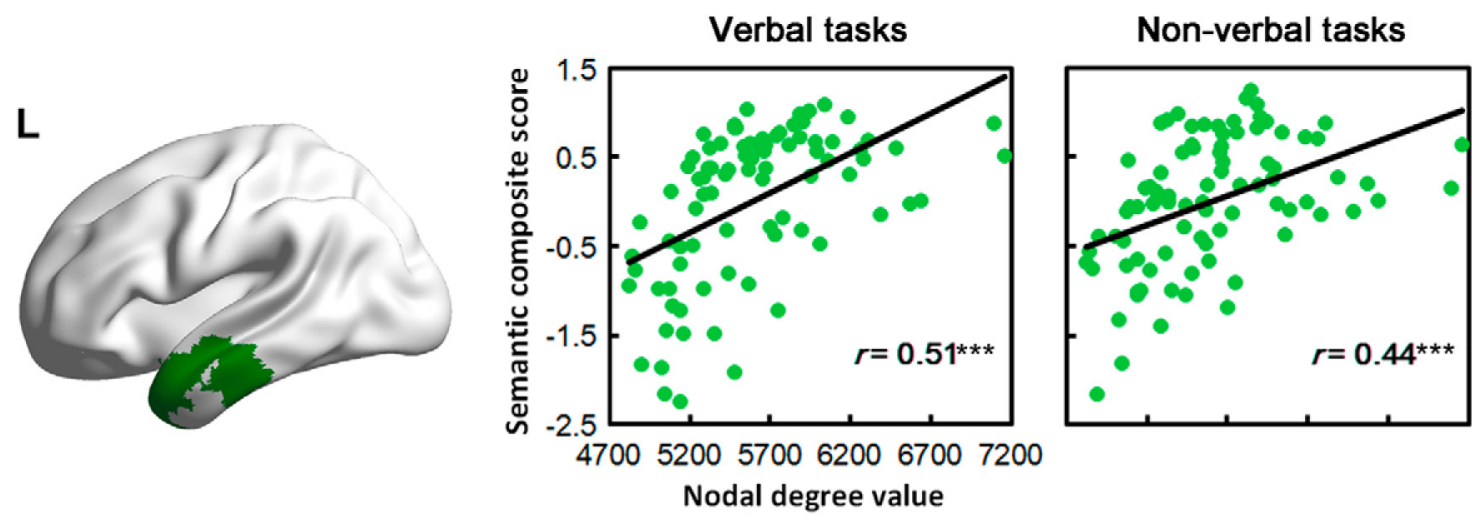

Nodal degree value
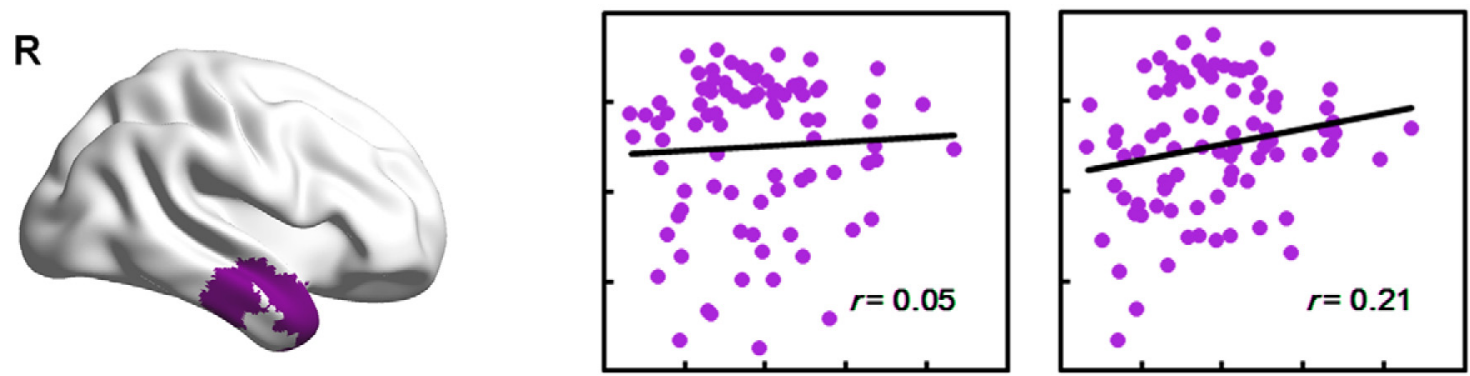

Figure 4. The correlations between nodal degree values of the bilateral anterior temporal lobes (ATLs) and semantic composite scores of verbal and nonverbal tasks. The brain maps in the left column depict the ATL regions (L, left; $R$, right), and the scatter plots in the right two columns show the correlational analysis results. ${ }^{* * *} p<0.001$.

(verbal semantics: $r=0.51, p<0.0001$; nonverbal semantics: $r=$ $0.44, p<0.0001 ;$ Fig. 4 ). When we additionally controlled for the influence of the three clinical variables (postonset month, etiology, total lesion voxels) and number calculation task among 80 patients, the correlations of the right ATL became insignificant with either type of task (verbal semantics: $r=0.01, p=0.91$; nonverbal semantics: $r=0.11, p=0.33$ ). However, those of the left ATL remained significant with both tasks (verbal semantics: 
$r=0.42, p<0.0001$; left ATL-nonverbal semantics: $r=0.30$, $p=0.007)$.

\section{Discussion}

Using rs-fMRI data and semantic behavioral data of 86 patients with brain damage, we investigated the hubs of the semantic network. We found that the left ATL and bilateral ACC could be such hubs because the functional connectivity strength of these cortices (measured by nodal degree values in the whole-brain network) could predict both general and modality-specific semantic processes. Our findings provide new supportive evidence for the distributed-plus-hub theories of the semantic system from the network perspective.

\section{Left anterior temporal lobe: a hub for semantic knowledge}

We found that the disconnection of the left ATL correlated with deficits in general and modality-specific semantic processing. This replicates the finding that the ATL is a semantic hub (Hodges et al., 1992; Garrard and Hodges, 2000; Hodges and Patterson, 2007; Patterson et al., 2007; Lambon Ralph et al., 2010; Pobric et al., 2010; Gorno-Tempini et al., 2011; Zhou et al., 2012; Lambon Ralph, 2014; Rice et al., 2015a,b; Chiou and Lambon Ralph, 2016). It might suggest that the left ATL is a convergent zone of semantic memory and that its connectivity with the rest of the brain embodies semantic knowledge.

Studies using various methodologies have consistently revealed the function of the ATL in semantic processing. For instance, white-matter fiber track studies and rs-fMRI studies have found that the ATL is widely connected with sensorimotorspecific and association cortices of the human brain (Binney et al., 2012; Guo et al., 2013; Fan et al., 2014; Pascual et al., 2015; Jackson et al., 2016). The atrophy and metabolism of the ATLs are associated with semantic impairments in semantic dementia (Butler et al., 2009; Mion et al., 2010). Transient dysfunctions of the ATL induced by repetitive transcranial magnetic stimulation caused longer reaction times on semantic tasks (Pobric et al., 2007, 2010; Lambon Ralph et al., 2009). The ATL was activated in semantic tasks in healthy subjects (Devlin et al., 2000; Visser and Lambon Ralph, 2011).

However, these studies mainly analyzed the regional properties of the ATL and connections from the ATL and, therefore, might not directly support the binding role of the ATL in the semantic system. Our study correlated the network properties (nodal degree) of the ATL with semantic performance and thus provided direct evidence for its hub role. Our results prove that the widely distributed connections of the ATL are indeed critical connections for maintaining semantic function and that the ATL is the hub region for these connections. Damaged connections with the ATL could cause deficits in the integration of semantic information.

\section{Bilateral anterior cingulate gyri: a hub for semantic control}

We also observed a significant correlation between the nodal degree of the ACC (extending to the medial prefrontal cortex) and semantic scores. One possible interpretation for this finding is that the ACC contributes to executive control in semantic processing. The ACC has been shown to be a part of the neocortex and is involved in various cognitive functions, such as emotional control, error recognition, and adaption to changes (Bush et al., 2000; Allman et al., 2001; Gasquoine, 2013). It was frequently activated in selective attention tasks and was thought to monitor distractive stimuli (Durston et al., 2003; Botvinick et al., 2004) and to estimate error rate (Botvinick et al., 1999). The ACC was also activated in semantic tasks, such as face recognition (Haxby et al., 1996) and semantic coding (Kapur et al., 1996). These findings suggest that the ACC is involved in the organization and executive control of semantic knowledge. Interestingly, functional connectivity of the ACC has a similar pattern with the temporal pole; both have wide connections with sensory and motor cortices (Margulies et al., 2007).

Our results might reflect the binding role of the ACC in semantic control. Through connections with sensory/motor cortices, the ACC could control which aspects of concepts to access; therefore, damaged connections with the ACC could cause deficits in semantic performance.

\section{Asymmetry of the left and right anterior temporal lobes}

We detected a semantic hub role for the left ATL, but detected no such role for the right ATL. This finding was inconsistent with the classical semantic distributed-plus-hub theory that the semantic hub includes both ATLs (Patterson et al., 2007). There are four potential possibilities to explain such lack of conformity with the right ATL: (1) This region is a semantic hub in a network. Our negative results might be due to the insensitive behavioral or brain imaging measurements. (2) The region is a local semantic region. Most prior studies considered the semantic role in local areas and found a semantic function of the right ATL (Patterson et al., 2007; Guo et al., 2013). However, our study investigated the function of this region from a network perspective, which resulted in null results. (3) This region is mainly responsible for abstract semantic processing (Rice et al., 2015a,b). Because our stimuli only included concrete objects, the effects of this region were not revealed. (4) The hub effects that previous studies observed might have been driven by the semantic nonverbal processing of this region (Gainotti, 2012; Mesulam et al., 2013; Hurley et al., 2015). This difference might reflect the mechanism for verbal/nonverbal dissociation in the left/right ATL.

As stated above, the semantic system in the human brain has at least two distinct modules: semantic knowledge of various objects and semantic control based on context (Jefferies et al., 2008; Whitney et al., 2012; Fang et al., 2015; Xu et al., 2016). The former is basic in the semantic system and is thus more frequently studied in the literature. The latter is also indispensable because we have abundant knowledge of objects; however, for a specific context, only relevant knowledge needs to be retrieved. For example, when boiling water in a high-altitude location, we need the knowledge that water boils at a lower temperature, whereas we do not need to know that it will release heat when mixed with a strong acid. The semantic control module works together with the knowledge module to accomplish semantic representation for our daily life.

\section{Limitations}

The current study has at least the following limitations. First, our semantic tasks only explored concrete concepts. The hubs of abstract semantics (e.g., social and emotional concepts) were not identified. This might be associated with the null results of the right ATL in our study. Second, the distribution of brain lesions in our patients was not equivalent across nodes and connections in the network, which led to different statistical powers for the analyses. Third, we only used the nodal degree value as the index of the neural network. It is not clear whether our findings could be replicated by adopting other cerebral network indexes (Rubinov and Sporns, 2010). Finally, our node degree measure pro- 
vided an average correlation with all other gray-matter voxels and reflected the general level of rs-fMRI correlations but not the pattern of these correlations. Thus, when node degree was correlated with semantic performance, it reflected the functional disconnection of the seed voxel but was not a measure of the functional integrity of the semantic network.

\section{Conclusion}

By correlating the nodal degree values of each voxel within the gray matter with the performance of general and modality-specific semantic processing in 86 patients, we identified two semantic hub regions: the left ATL and the bilateral ACC. The reduction of nodal degree values of the regions was significantly correlated with the deterioration in semantic performance of the patients. These regions play a critical role in the semantic knowledge subnetwork and the semantic control subnetwork, respectively. Our findings provide empirical evidence for the distributed-plus-hub theory of the semantic system.

\section{References}

Allman JM, Hakeem A, Erwin JM, Nimchinsky E, Hof P (2001) The anterior cingulate cortex. The evolution of an interface between emotion and cognition. Ann N Y Acad Sci 935:107-117. Medline

Barsalou LW (2008) Grounded cognition. Annu Rev Psychol 59:617-645. CrossRef Medline

Bi Y, Han Z, Zhong S, Ma Y, Gong G, Huang R, Song L, Fang Y, He Y, Caramazza A (2015) The white matter structural network underlying human tool use and tool understanding. J Neurosci 35:6822-6835. CrossRef Medline

Binder JR, Desai RH (2011) The neurobiology of semantic memory. Trends Cogn Sci 15:527-536. CrossRef Medline

Binder JR, Desai RH, Graves WW, Conant LL (2009) Where is the semantic system? A critical review and meta-analysis of 120 functional neuroimaging studies. Cereb Cortex 19:2767-2796. CrossRef Medline

Binney RJ, Embleton KV, Jefferies E, Parker GJ, Ralph MA (2010) The ventral and inferolateral aspects of the anterior temporal lobe are crucial in semantic memory: evidence from a novel direct comparison of distortion-corrected fMRI, rTMS, and semantic dementia. Cereb Cortex 20:2728-2738. CrossRef Medline

Binney RJ, Parker GJ, Lambon Ralph MA (2012) Convergent connectivity and graded specialization in the rostral human temporal lobe as revealed by diffusion-weighted imaging probabilistic tractography. J Cogn Neurosci 24:1998-2014. CrossRef Medline

Bonnici HM, Richter FR, Yazar Y, Simons JS (2016) Multimodal feature integration in the angular gyrus during episodic and semantic retrieval. J Neurosci 36:5462-5471. CrossRef Medline

Botvinick M, Nystrom LE, Fissell K, Carter CS, Cohen JD (1999) Conflict monitoring versus selection-for-action in anterior cingulate cortex. Nature 402:179-181. CrossRef Medline

Botvinick MM, Cohen JD, Carter CS (2004) Conflict monitoring and anterior cingulate cortex: an update. Trends Cogn Sci 8:539-546. CrossRef Medline

Bush G, Luu P, Posner MI (2000) Cognitive and emotional influences in anterior cingulate cortex. Trends Cogn Sci 4:215-222. CrossRef Medline

Butler CR, Brambati SM, Miller BL, Gorno-Tempini ML (2009) The neural correlates of verbal and non-verbal semantic processing deficits in neurodegenerative disease. Cogn Behav Neurol 22:73-80. CrossRef Medline

Chao-Gan Y, Yu-Feng Z (2010) DPARSF: a MATLAB toolbox for" pipeline" data analysis of resting-state fMRI. Front Syst Neurosci 4:13. Medline

Chiou R, Lambon Ralph MA (2016) The anterior temporal cortex is a primary semantic source of top-down influences on object recognition. Cortex 79:75-86. CrossRef Medline

Crawford JR, Garthwaite PH (2006) Comparing patients' predicted test scores from a regression equation with their obtained scores: a significance test and point estimate of abnormality with accompanying confidence limits. Neuropsychology 20:259-271. CrossRef Medline

Devlin JT, Russell RP, Davis MH, Price CJ, Wilson J, Moss HE, Matthews PM, Tyler LK (2000) Susceptibility-induced loss of signal: comparing PET and fMRI on a semantic task. Neuroimage 11:589-600. CrossRef Medline
Durston S, Davidson MC, Thomas KM, Worden MS, Tottenham N, Martinez A, Watts R, Ulug AM, Casey BJ (2003) Parametric manipulation of conflict and response competition using rapid mixed-trial event-related fMRI. Neuroimage 20:2135-2141. CrossRef Medline

Fan L, Wang J, Zhang Y, Han W, Yu C, Jiang T (2014) Connectivity-based parcellation of the human temporal pole using diffusion tensor imaging. Cereb Cortex 24:3365-3378. CrossRef Medline

Fang Y, Han Z, Zhong S, Gong G, Song L, Liu F, Huang R, Du X, Sun R, Wang $\mathrm{Q}, \mathrm{He} \mathrm{Y}, \mathrm{Bi} \mathrm{Y}$ (2015) The semantic anatomical network: evidence from healthy and brain-damaged patient populations. Hum Brain Mapp 36: 3499-3515. CrossRef Medline

Forster KI, Forster JC (2003) DMDX: a windows display program with millisecond accuracy. Behav Res Methods Instrum Comput 35:116-124. CrossRef Medline

Gainotti G (2012) The format of conceptual representations disrupted in semantic dementia: a position paper. Cortex 48:521-529. CrossRef Medline

Garrard P, Hodges JR (2000) Semantic dementia: clinical, radiological and pathological perspectives. J Neurol 247:409-422. CrossRef Medline

Gasquoine PG (2013) Localization of function in anterior cingulate cortex: from psychosurgery to functional neuroimaging. Neurosci Biobehav Rev 37:340-348. CrossRef Medline

Gorno-Tempini ML, Hillis AE, Weintraub S, Kertesz A, Mendez M, Cappa SF, Ogar JM, Rohrer JD, Black S, Boeve BF, Manes F, Dronkers NF, Vandenberghe R, Rascovsky K, Patterson K, Miller BL, Knopman DS, Hodges JR, Mesulam MM, Grossman M (2011) Classification of primary progressive aphasia and its variants. Neurology 76:1006-1014. CrossRef Medline

Guo CC, Gorno-Tempini ML, Gesierich B, Henry M, Trujillo A, Shany-Ur T, Jovicich J, Robinson SD, Kramer JH, Rankin KP, Miller BL, Seeley WW (2013) Anterior temporal lobe degeneration produces widespread network-driven dysfunction. Brain 136:2979-2991. CrossRef Medline

Han Z, Bi Y, Chen J, Chen Q, He Y, Caramazza A (2013a) Distinct regions of right temporal cortex are associated with biological and human-agent motion: functional magnetic resonance imaging and neuropsychological evidence. J Neurosci 33:15442-15453. CrossRef Medline

Han Z, Ma Y, Gong G, He Y, Caramazza A, Bi Y (2013b) White matter structural connectivity underlying semantic processing: evidence from brain damaged patients. Brain 136:2952-2965. CrossRef Medline

Haxby JV, Ungerleider LG, Horwitz B, Maisog JM, Rapoport SI, Grady CL (1996) Face encoding and recognition in the human brain. Proc Natl Acad Sci U S A 93:922-927. CrossRef Medline

Hodges JR, Patterson K (2007) Semantic dementia: a unique clinicopathological syndrome. Lancet Neurol 6:1004-1014. CrossRef Medline

Hodges JR, Patterson K, Oxbury S, Funnell E (1992) Semantic dementia. Brain 115:1783-1806. CrossRef Medline

Hoffman P, Evans GA, Lambon Ralph MA (2014) The anterior temporal lobes are critically involved in acquiring new conceptual knowledge: evidence for impaired feature integration in semantic dementia. Cortex 50 : 19-31. CrossRef Medline

Howard D, Patterson KE (1992) The Pyramids and Palm Trees Test: a test of semantic access from words and pictures. Bury St. Edmunds, UK: Thames Valley Test Company.

Hurley RS, Bonakdarpour B, Wang X, Mesulam MM (2015) Asymmetric connectivity between the anterior temporal lobe and the language network. J Cogn Neurosci 27:464-473. CrossRef Medline

Huth AG, de Heer WA, Griffiths TL, Theunissen FE, Gallant JL (2016) Natural speech reveals the semantic maps that tile human cerebral cortex. Nature 532:453-458. CrossRef Medline

Jackson RL, Hoffman P, Pobric G, Lambon Ralph MA (2016) The semantic network at work and rest: differential connectivity of anterior temporal lobe subregions. J Neurosci 36:1490-1501. CrossRef Medline

Jefferies E, Lambon Ralph MA (2006) Semantic impairment in stroke aphasia versus semantic dementia: a case-series comparison. Brain 129:21322147. CrossRef Medline

Jefferies E, Patterson K, Ralph MA (2008) Deficits of knowledge versus executive control in semantic cognition: insights from cued naming. Neuropsychologia 46:649-658. CrossRef Medline

Kapur S, Tulving E, Cabeza R, McIntosh AR, Houle S, Craik FI (1996) The neural correlates of intentional learning of verbal materials: a PET study in humans. Brain Res Cogn Brain Res 4:243-249. CrossRef Medline

Lambon Ralph MA (2014) Neurocognitive insights on conceptual knowl- 
edge and its breakdown. Philos Trans R Soc Lond B Biol Sci 369: 20120392. CrossRef Medline

Lambon Ralph MA, Pobric G, Jefferies E (2009) Conceptual knowledge is underpinned by the temporal pole bilaterally: convergent evidence from rTMS. Cereb Cortex 19:832-838. CrossRef Medline

Lambon Ralph MA, Sage K, Jones RW, Mayberry EJ (2010) Coherent concepts are computed in the anterior temporal lobes. Proc Natl Acad Sci U S A 107:2717-2722. CrossRef Medline

Lambon Ralph MA, Ehsan S, Baker GA, Rogers TT (2012) Semantic memory is impaired in patients with unilateral anterior temporal lobe resection for temporal lobe epilepsy. Brain 135:242-258. CrossRef Medline

Mahon BZ, Caramazza A (2009) Concepts and categories: a cognitive neuropsychological perspective. Annu Rev Psychol 60:27-51. CrossRef Medline

Margulies DS, Kelly AM, Uddin LQ, Biswal BB, Castellanos FX, Milham MP (2007) Mapping the functional connectivity of anterior cingulate cortex. Neuroimage 37:579-588. CrossRef Medline

Martin A (2007) The representation of object concepts in the brain. Annu Rev Psychol 58:25-45. CrossRef Medline

Martin A (2016) GRAPES-Grounding representations in action, perception, and emotion systems: how object properties and categories are represented in the human brain. Psychon Bull Rev 23:979-990. CrossRef Medline

Martin A, Kyle Simmons W, Beauchamp MS, Gotts SJ (2014) Is a single "hub," with lots of spokes, an accurate description of the neural architecture of action semantics? Comment on "Action semantics: a unifying conceptual framework for the selective use of multimodal and modalityspecific object knowledge" by van Elk, Van Schie and Bekkering. Phys Life Rev 11:261-262. CrossRef Medline

Mesulam MM, Wieneke C, Hurley R, Rademaker A, Thompson CK, Weintraub S, Rogalski EJ (2013) Words and objects at the tip of the left temporal lobe in primary progressive aphasia. Brain 136:601-618. CrossRef Medline

Mion M, Patterson K, Acosta-Cabronero J, Pengas G, Izquierdo-Garcia D, Hong YT, Fryer TD, Williams GB, Hodges JR, Nestor PJ (2010a) What the left and right anterior fusiform gyri tell us about semantic memory. Brain 133:3256-3268. CrossRef Medline

Oldfield RC (1971) The assessment and analysis of handedness: the Edinburgh inventory. Neuropsychologia 9:97-113. CrossRef Medline

Pascual B, Masdeu JC, Hollenbeck M, Makris N, Insausti R, Ding SL, Dickerson BC (2015) Large-scale brain networks of the human left temporal pole: a functional connectivity MRI study. Cereb Cortex 25:680-702. CrossRef Medline

Patterson K, Nestor PJ, Rogers TT (2007) Where do you know what you know? The representation of semantic knowledge in the human brain. Nat Rev Neurosci 8:976-987. Medline

Patterson K, Kopelman MD, Woollams AM, Brownsett SL, Geranmayeh F, Wise RJ (2015) Semantic memory: which side are you on? Neuropsychologia 76:182-191. CrossRef Medline

Peelen MV, Caramazza A (2012) Conceptual object representations in human anterior temporal cortex. J Neurosci 32:15728-15736. CrossRef Medline

Pobric G, Jefferies E, Ralph MA (2007) Anterior temporal lobes mediate semantic representation: mimicking semantic dementia by using rTMS in normal participants. Proc Natl Acad Sci U S A 104:20137-20141. CrossRef Medline

Pobric G, Jefferies E, Ralph MA (2010) Amodal semantic representations depend on both anterior temporal lobes: evidence from repetitive transcranial magnetic stimulation. Neuropsychologia 48:1336-1342. CrossRef Medline

Power JD, Barnes KA, Snyder AZ, Schlaggar BL, Petersen SE (2012) Spurious but systematic correlations in functional connectivity MRI networks arise from subject motion. Neuroimage 59:2142-2154. CrossRef Medline

Rice GE, Hoffman P, Lambon Ralph MA (2015a) Graded specialization within and between the anterior temporal lobes. Ann N Y Acad Sci 1359: 84-97. CrossRef Medline

Rice GE, Lambon Ralph MA, Hoffman P (2015b) The roles of left versus right anterior temporal lobes in conceptual knowledge: an ALE metaanalysis of 97 functional neuroimaging studies. Cereb Cortex 25:43744391. CrossRef Medline

Rubinov M, Sporns O (2010) Complex network measures of brain connectivity: uses and interpretations. Neuroimage 52:1059-1069. CrossRef Medline

Seghier ML (2013) The angular gyrus multiple functions and multiple subdivisions. Neuroscientist 19:43-61. CrossRef Medline

Shimotake A, Matsumoto R, Ueno T, Kunieda T, Saito S, Hoffman P, Kikuchi T, Fukuyama H, Miyamoto S, Takahashi R, Ikeda A, Lambon Ralph MA (2015) Direct exploration of the role of the ventral anterior temporal lobe in semantic memory: cortical stimulation and local field potential evidence from subdural grid electrodes. Cereb Cortex 25:3802-3817.

Tsapkini K, Frangakis CE, Hillis AE (2011) The function of the left anterior temporal pole: evidence from acute stroke and infarct volume. Brain 134: 3094-3105. CrossRef Medline

Tulving E (1972) Episodic and semantic memory. In: Organization of memory (Tulving E, Donaldson W, eds), pp 381-403. London: Academic.

Tzourio-Mazoyer N, Landeau B, Papathanassiou D, Crivello F, Etard O, Delcroix N, Mazoyer B, Joliot M (2002) Automated anatomical labeling of activations in SPM using a macroscopic anatomical parcellation of the MNI MRI single-subject brain. Neuroimage 15:273-289. CrossRef Medline

Visser M, Lambon Ralph MA (2011) Differential contributions of bilateral ventral anterior temporal lobe and left anterior superior temporal gyrus to semantic processes. J Cogn Neurosci 23:3121-3131. CrossRef Medline

Visser M, Jefferies E, Lambon Ralph MA (2010) Semantic processing in the anterior temporal lobes: a meta-analysis of the functional neuroimaging literature. J Cogn Neurosci 22:1083-1094. CrossRef Medline

Wang J, Wang X, Xia M, Liao X, Evans A, He Y (2015) GRETNA: a graph theoretical network analysis toolbox for imaging connectomics. Front Hum Neurosci 9:386. CrossRef Medline

Wei T, Liang X, He Y, Zang Y, Han Z, Caramazza A, Bi Y (2012) Predicting conceptual processing capacity from spontaneous neuronal activity of the left middle temporal gyrus. J Neurosci 32:481-489. CrossRef Medline

Whitney C, Kirk M, O’Sullivan J, Lambon Ralph MA, Jefferies E (2012) Executive semantic processing is underpinned by a large-scale neural network: revealing the contribution of left prefrontal, posterior temporal, and parietal cortex to controlled retrieval and selection using TMS. J Cogn Neurosci 24:133-147. CrossRef Medline

Xu Y, Lin Q, Han Z, He Y, Bi Y (2016) Intrinsic functional network architecture of human semantic processing: modules and hubs. Neuroimage 132:542-555. CrossRef Medline

Zhou J, Gennatas ED, Kramer JH, Miller BL, Seeley WW (2012) Predicting regional 652 neurodegeneration from the healthy brain functional connectome. Neuron 73:1216-1227. CrossRef Medline 